\title{
Immediate vs delayed sequential cataract surgery: a comparative study
}

N Nassiri' ${ }^{1}$ N Nassiri ${ }^{2}$, SH Sadeghi Yarandi ${ }^{2}$ and M Rahnavardi ${ }^{3}$

\begin{abstract}
Purpose Immediate sequential cataract surgery (ISCS) is still a controversial procedure. We compared the clinical and patient-reported outcomes of ISCS $v s$ the usual delayed sequential cataract surgery
\end{abstract} (DSCS).

Methods Selected patients with bilateral cataracts were assigned nonrandomly and according to their preferences to either ISCS in one session (intervention group) or DSCS over two sessions with a 2-month interval between sessions (control group). Binocular visual acuity, binocular contrast sensitivity, stereopsis, and self-reported visual function (VF-14) were measured preoperatively and postoperatively for up to 4 months after the second-eye surgery. Mann-Whitney $U$-test was used to compare quantitative variables, while the $\chi^{2}$ and Fisher's exact tests were used for qualitative variables.

Results Of the 220 eligible patients, 74 (33.6\%) chose ISCS, and 137 (62.3\%) selected DSCS. The remaining patients $(4.1 \%)$ were allocated randomly to either group. For 2 months, the outcome measures were significantly worse in the DSCS group than in the ISCS group. However, after 4 months (2 and 4 months after second-eye surgery in the DSCS and ISCS groups, respectively) the differences became insignificant except for VF-14 $(P<0.05)$. The mean post-operative objective measures and their differences from baseline were not significantly different between the groups. Nevertheless, postoperatively, VF-14 improved more $(P<0.05)$ and attained a higher value $(P<0.05)$ in the ISCS group.

Conclusions In experienced hands, with stringent patient selection criteria and with a strict aseptic protocol, ISCS can safely provide a more rapid rehabilitation of VF than DSCS. Eye (2009) 23, 89-95; doi:10.1038/sj.eye.6702989; published online 5 October 2007
Keywords: bilateral cataract surgery; immediate sequential cataract surgery; simultaneous bilateral cataract surgery; delayed sequential cataract surgery

\section{Introduction}

Immediate sequential cataract surgery (ISCS), also referred to as simultaneous bilateral cataract surgery, is still a controversial procedure. An increasing number of sources recommend that cataract surgery should be done for both eyes in patients with bilateral cataracts. ${ }^{1-7}$ However, it is still unknown whether it is better for these patients to have ISCS or delayed sequential cataract surgery (DSCS). Despite the obvious benefits of ISCS to patients and society, ${ }^{8-11}$ the major reluctance of ophthalmologists to perform ISCS revolves to some extent around its non-catastrophic complications, such as refractive surprises, and most importantly, its catastrophic complications, such as endophthalmitis, ${ }^{11}$ especially due to contaminated instrument-derived incidents; ${ }^{12}$ however, the literature indicates that the safety of ISCS has been increasing, ${ }^{1,13-17}$ particularly with recent advancements of microsurgical techniques ${ }^{18,19}$ and the use of strict protocols for post-operative endophthalmitis prophylaxis. ${ }^{20}$ Furthermore, if an intraoperative complication occurs during the first-eye surgery, surgery for the second eye can be aborted.

In this trial, the clinical- and patient-reported outcomes, as well as intraoperative and postoperative complications, were compared between patients who underwent ISCS and DSCS with a 2-month interval between surgeries.

Materials and methods

\section{Patients}

A prospective, nonrandomized clinical trial was performed from January 2004 to September 2005
${ }^{1}$ Department of Ophthalmology, Imam Hossein Medical Center, Shaheed Beheshti University of Medical Sciences, Tehran, Iran

${ }^{2}$ Vanak Eye Surgery Center, Tehran, Iran

${ }^{3}$ Didegan-Salamat Research Institute, Tehran, Iran

Correspondence: N Nassiri, Department of Ophthalmology, Vanak Eye Surgery Center, No 41, Haghani highway, Vanak Square, Tehran 15948, Iran Tel: $(+98) 2188774143$, (+98) 218877 6521; Fax: (+98) 2188773605 . E-mail: drnassirin@ sbmu.ac.ir

Received: 4 May 2007 Accepted in revised form: 16 August 2007 Published online: 5 October 2007

The article has not been presented in a meeting 
at the Vanak Eye Surgery Center, Tehran, Iran. All operations were performed by the same experienced surgeon (Nader N) with a 5-year mean of 320 cataract operations annually. All consecutive patients with bilateral, visually significant cataract were eligible to enter the trial. Exclusion criteria were increased risk of endophthalmitis (eg, active adenexal and ocular surface infection, immunosuppression and immunodeficiency, systemic steroids, diabetes mellitus), axial extremes (axial length $>27$ or $<21 \mathrm{~mm}$ ), posterior segment disorders, preexisting ocular comorbidities (eg, pterygium, glaucoma, phacodonesis, and corneal opacity), history of previous ocular surgeries (eg, trabeculectomy, refractive surgery), history of ocular trauma, and intraoperative complications during the first-eye surgery (eg, vitreous loss, posterior capsule rupture, very prolonged surgery due to surgical difficulties). The same trained consultant discussed the known advantages and disadvantages of ISCS and DSCS with all patients and their families; handouts were given to all patients. The patients were free to choose either ISCS (intervention group) or DSCS (control group). Those who opted for ISCS were asked to explain their rationale by prioritizing six proposed reasons. Ambivalent patients who could not select their surgery $(n=9)$ were assigned to either group by a coin toss procedure. In the control group, patients had surgery on the first eye within the first month after enrolling in the study. Subsequently, surgery was carried out on the second eye 2 months later. Patients in the ISCS had simultaneous surgery of both eyes within 1 month after their enrollment; they were informed about the possibility of the second-eye surgery being aborted if an intraoperative complication occurred during surgery on the first eye. All recruited patients gave their written informed consent, and the study was performed in accordance with the Declaration of Helsinki. We certify that all applicable institutional and governmental regulations concerning the ethical use of human subjects were followed during this research.

\section{Assessments}

Preoperative and follow-up comprehensive systemic and ocular examinations were carried out by the same surgeon and two trained optometrists. Presurgical assessments included slit-lamp examination and ophthalmoloscopy. As well, visual acuity, binocular contrast sensitivity, stereopsis, intraocular pressure, keratometry, and axial length were assessed. Three clinical measures, including binocular visual acuity, binocular contrast sensitivity, and stereopsis, and a patient-reported visual disability score, the VF-14, were the main outcome measures; these were assessed preoperatively (baseline) and postoperatively for up to 4 months after the last-eye surgery.

The logMAR best-corrected visual acuity was measured with a Snellen chart calibrated for a $20-\mathrm{ft}$ ( $\sim 6 \mathrm{~m}$ ) distance using the line assignment method. Refraction between the left eye (LE) and the right eye (RE) was calculated using the spherical equivalent. Binocular contrast sensitivity was measured using Pelli-Robson charts at $1 \mathrm{~m} \cdot{ }^{21-23}$ Patients who had difficulties with Latin letters were familiarized with the letters before the exam, and rarely, they were asked to draw what they saw. Stereopsis was assessed using the TNO test. Lower visual acuity and stereopsis values and higher contrast sensitivity values were suggestive of good vision.

The VF-14 was used to measure patient-reported visual disability. ${ }^{24}$ The VF-14 is a 14-item scale, based on 14 everyday activities that are the most often reported measures of functional ability. The VF-14 score ranges from 0 (maximum disability) to 100 (no disability). It has been extensively validated internationally and is highly reliable, reproducible, valid, internally consistent, and responsive to clinically significant changes. ${ }^{25-26}$

\section{Operation}

All surgeries were carried out under topical anaesthesia in an outpatient setting. The standard procedure included phacoemulsification (Advanced Medical Optics, Sovereign ${ }^{\circledR}$ system with WhiteStar ${ }^{\mathrm{TM}}$ Technology, Santa Ana, CA, USA) with pupil dilation using tropicamide $1 \%$ and phenylephrine $5 \%$, topical anaesthesia using tetracaine hydrochloride $0.5 \%$, a 3.2-mm, clear, corneal incision plus a second paracentesis, and implantation of a foldable acrylic posterior chamber intraocular lens (Alcon, Fort Worth, TX, USA); no sutures were used. Infection prophylaxis included preoperative topical ciprofloxacin (Ciloxan ${ }^{\circledR} 0.3 \%$, Alcon Laboratories (UK) Limited), povidone-iodine $10 \%$, intracameral administration of $1 \mathrm{mg}$ vancomycin hydrochloride (Vancocin ${ }^{\circledR}$, Eli Lilly and Company, Indianapolis, IN, USA), $10 \mathrm{mg} / \mathrm{ml}$, and post-operative topical ciprofloxacin. Patients in the control group had serial operations (DSCS) with a 2-month interval between them. The patients in the ISCS group had simultaneous surgeries on both eyes in one session (ISCS); the surgeons and the assistants resterilized their hands and regowned for the second-eye surgery, which was considered to be a completely separate procedure. The second eye was then prepped and draped, and a separate set of surgical instruments, irrigating lines, and fluids were used; however, the same phacomachine was used. In both groups postoperatively, the antibiotic (topical 
ciprofloxacin) was discontinued after 1 week, and the dexamethasone was tapered within 4 weeks.

\section{Statistical analysis}

Baseline demographic factors and outcome measures were compared. The two groups' post-operative values obtained during the second and fourth months of followup were compared. The mean post-operative values 2 and 4 months after the last surgery were calculated for both groups. The means of the outcome measures and their difference from their baseline values were compared. The nonparametric Mann-Whitney $U$-test was used to compare quantitative variables, while the $\chi^{2}$ and Fisher's exact tests were used for qualitative variables. The patient-ranked reasons for selecting ISCS were analysed by calculating the Friedman mean rank score; the differences among the reasons were checked by the Friedman nonparametric test. Statistical significance was set at $P<0.05$.

\section{Results}

Of the 294 patients who were assessed, 220 (74.8\%) eligible patients were enrolled. Seventy-four (33.6\%) patients chose ISCS (intervention group), whereas 137 $(62.3 \%)$ selected DSCS (control group). Nine (4.1\%) ambivalent patients were allocated by coin toss: 6 to the ISCS group and 3 to the DSCS group. Ten patients in the intervention group (12.5\%) and 26 in the control group (18.6\%) did not complete the trial; this difference was not significant. Figure 1 shows the study flow chart.

The mean age of the study participants was $66.84 \pm 6.21$ (range 54-83) years and 119 were women (54.1\%). Eightynine $(40.5 \%)$ had a higher education (Bachelor of Science or above). Baseline values were similar in the two study groups (Table 1).

In the ISCS group, the proposed six reasons for selecting ISCS were given significantly different ranks by the study subjects $(P<0.001)$; 'rapid improvement of eyesight' was the first reason chosen (Table 2).

Compared to the DSCS group, all subjective and objective outcome measures were significantly better in the ISCS group 2 months after the primary operation. However, these differences became insignificant 2 months later (month 4), although the difference in visual disability remained significant (Table 3 ).

Two months after the first-eye surgery, anisometropia $(\geqslant 2.0 \mathrm{D})$ was detected in 41 patients $(36 \%)$ in the DSCS group and in none of the ISCS group. Furthermore, the insignificant baseline refraction difference between the DSCS group and the ISCS group became significant in month $2(P<0.001)$, but again became insignificant 4 months after the first-eye surgery (Table 3 ).

Ninety per cent of patients in the ISCS group and $87 \%$ of patients in the DSCS group had a mean visual acuity of $\leqslant 0.1 \log$ MAR units within 4 months after the first-eye surgery. The VF-14 for visual disability improved

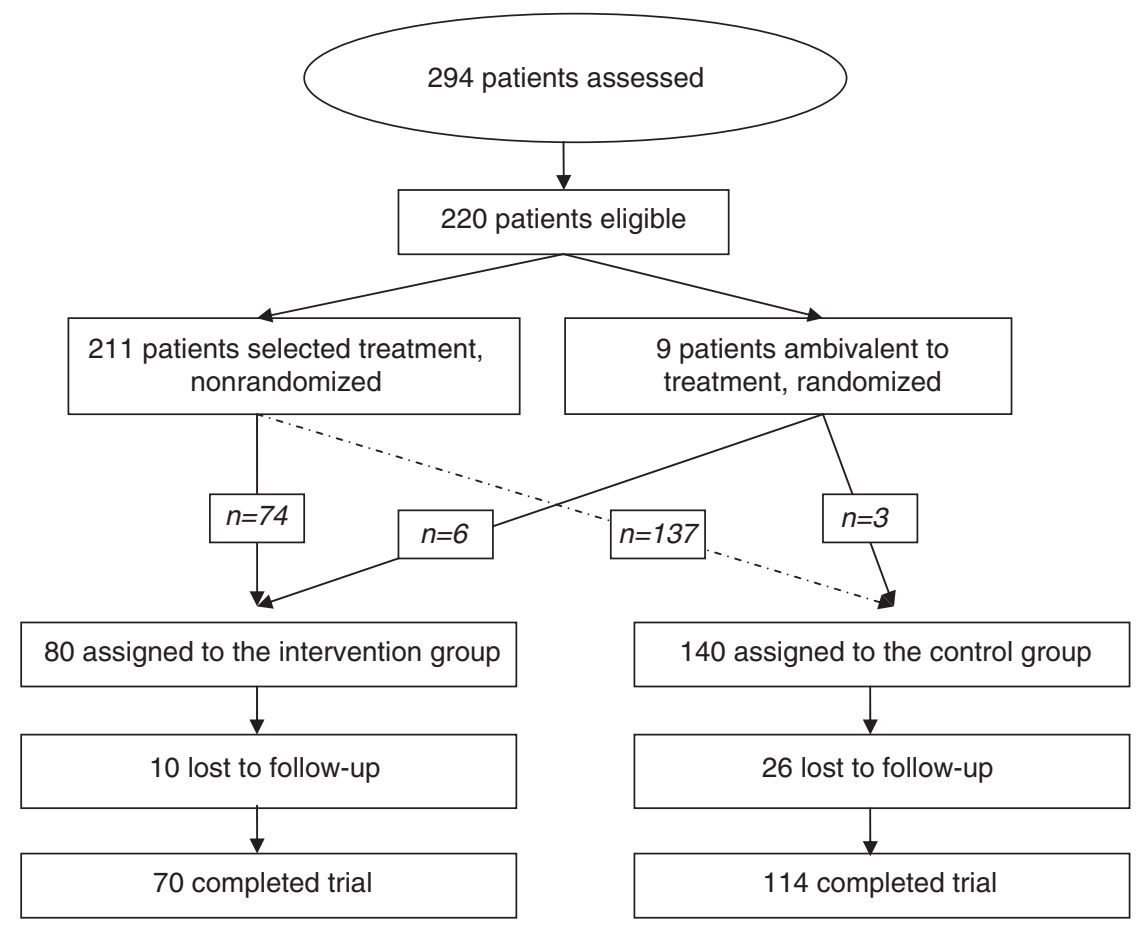

Figure 1 Trial flow chart. 
Table 1 Baseline values of the ISCS and DSCS groups

\begin{tabular}{|c|c|c|}
\hline & $\begin{array}{c}\text { ISCS: } \\
\text { intervention, } \\
\mathrm{n}=80\end{array}$ & $\begin{array}{l}\text { DSCS: control, } \\
\quad \mathrm{n}=140\end{array}$ \\
\hline \multicolumn{3}{|l|}{ Demographic characteristics } \\
\hline Age, years, mean (SD) & $67.31(7.2)$ & $66.56(5.6)$ \\
\hline Age $\geqslant 65$ years, $n(\%)$ & $51(63.7)$ & 89 (63.6) \\
\hline Gender, female, $n(\%)$ & $44(55.0)$ & $75(53.6)$ \\
\hline Education, below BS, $n(\%)$ & $42(52.5)$ & $89(63.6)$ \\
\hline \multicolumn{3}{|l|}{ Objective measures } \\
\hline $\begin{array}{l}\text { Binocular visual acuity, } \\
\text { logMAR units, median (IQR) }\end{array}$ & $0.80(0.30)$ & $0.80(0.40)$ \\
\hline $\begin{array}{l}\text { Binocular contrast } \\
\text { sensitivity }{ }^{\mathrm{a}} \text {, median (IQR) }\end{array}$ & $1.20(0.30)$ & $1.20(0.44)$ \\
\hline Stereopsis ${ }^{\mathrm{b}}$, median (IQR) & $400(660)$ & $400(660)$ \\
\hline Refraction difference & $0.25(0.50)$ & $0.25(0.50)$ \\
\hline \multicolumn{3}{|l|}{$\begin{array}{l}\text { between RE and LE, D, } \\
\text { median (IQR) }\end{array}$} \\
\hline$\geqslant 2, n(\%)$ & $6(7.5)$ & $15(10.7)$ \\
\hline \multicolumn{3}{|l|}{ Subjective measure } \\
\hline $\begin{array}{l}\text { Visual disability (VF-14) } \\
\text { score, median (IQR) }\end{array}$ & $39.6(24.6)$ & $42.1(25.4)$ \\
\hline \multicolumn{3}{|c|}{$\begin{array}{l}\mathrm{BS} \text {, Bachelor of Science; } \mathrm{D} \text {, Diopter; DSCS, delayed sequential cataract } \\
\text { surgery; ISCS, immediate sequential cataract surgery; IQR, inter-quartile } \\
\text { range; LE, left eye; RE, right eye. }\end{array}$} \\
\hline
\end{tabular}

Table 2 Patient-ranked reasons for selecting immediate sequential cataract surgery ${ }^{\mathrm{a}}$

\begin{tabular}{lc}
\hline Reason & $\begin{array}{c}\text { Rank (Friedman } \\
\text { mean rank) }\end{array}$ \\
\hline Rapid improvement of eyesight & $1(2.0)$ \\
Need to have surgery only once & $2(3.1)$ \\
Reduced number of visits & $3(3.6)$ \\
Reduced total rehabilitation time & $4(3.9)$ \\
Time limitation & $5(3.9)$ \\
Difficulties traveling & $6(4.5)$
\end{tabular}

${ }^{\mathrm{a}} \chi^{2}=67.92$, d.f. $=5, P<0.001$ for Friedman test.

significantly more in the ISCS patients than in the DSCS group $(P<0.05$; Table 4$)$.

Within 4 months after the second-eye surgery, the following complications were noted in the ISCS and DSCS groups: posterior capsule rupture (1 vs 0 eyes), hyphema (1 vs 4 eyes), cystoid macular oedema (2 vs 1 eyes), anterior uveitis ( 1 vs 3 eyes), raised

IOP $>22 \mathrm{mmHg}(0$ vs 3$)$, and IOL power error $>1.00 \mathrm{D}$ ( 2 vs 2 eyes).

\section{Discussion}

In this study, in accordance with ethical considerations, patients were free to choose either DSCS or ISCS. Most patients $(62.3 \%)$ preferred the well-known DSCS, although those in favour of ISCS ranked rapid improvement of their eyesight as the most important reason for their selecting ISCS. Since this study was nonrandomized, the patients were not equally distributed between the study groups. Nevertheless, both groups had similar baseline characteristics, and nonparametric methods were used for the statistical analysis to provide an unbiased evaluation of the intervention.

Compared to DSCS, ISCS offered faster improvement of visual function (VF). The remaining opacity in the non-operated eye of the DSCS group patients led to poorer VF indices, binocular visual acuity, binocular contrast sensitivity, stereopsis, and patient-reported visual disability in month 2 before the second-eye surgery was performed. In practice, this delay would be much longer on a typical surgical waiting list. During this waiting period, these patients would have suboptimal VF and visual-related quality of life.

However, no surveys have been carried out to determine whether suboptimal VF improves over time as a result of patient adaptation.

In the DSCS group, the second-eye surgery improved binocular visual acuity by one Snellen line; however, previous studies have shown only marginal improvement in visual acuity after second-eye surgery. ${ }^{3,5,6,27}$ Similarly, second-eye surgery caused improvement in contrast sensitivity and stereopsis, respectively. A considerable improvement, greater than the 5-point change that has been suggested to be clinically significant, was observed in patient-reported visual disability (VF-14). ${ }^{2}$ Lundström $e t$ al ${ }^{1}$ similarly reported that ISCS patients had more rapid rehabilitation than DSCS patients with respect to binocular visual acuity and binocular contrast sensitivity but not in stereopsis.

Four months after the last-eye surgery (month 4 for ISCS and month 6 for DSCS), the mean post-operative outcome measures were not significantly different between the groups for the objective measures, but they were improved for VF-14, which is a subjective measure. Lundström $e a^{1}{ }^{1}$ similarly mentioned that the long-term benefits did not differ between the two groups.

The VF-14 questionnaire is a reliable, valid index of a patient's ability to perform 14 visual activities. ${ }^{24}$ We used this questionnaire and found it to be the only outcome measure that remained significantly higher postoperatively in the ISCS group compared to the DSCS group, not only 2 months, but also 4 months after the first operation. Since the difference remained significant after 4 months only in the subjective measure (VF-14) and not in the objective measures, we can hypothesize that patients in the ISCS group may have been more socially active and may have had more subjective feelings about 
Table 3 Postoperative measures obtained 2 and 4 months after the first surgery ${ }^{\mathrm{a}}$

\begin{tabular}{|c|c|c|c|c|c|c|}
\hline \multirow[t]{2}{*}{ Measure } & \multicolumn{3}{|c|}{ After 2 months } & \multicolumn{3}{|c|}{ After 4 months } \\
\hline & $\begin{array}{l}\text { Intervention } \\
\text { group, } \mathrm{n}=70\end{array}$ & $\begin{array}{l}\text { Control group } \\
\mathrm{n}=114\end{array}$ & $\mathrm{P}-$ value $^{\mathrm{b}}$ & $\begin{array}{l}\text { Intervention } \\
\text { group, } \mathrm{n}=70\end{array}$ & $\begin{array}{c}\text { Control group, } \\
\mathrm{n}=114\end{array}$ & $\mathrm{P}-$-value ${ }^{\mathrm{b}}$ \\
\hline $\begin{array}{l}\text { Binocular visual acuity, logMAR } \\
\text { units, median (IQR) }\end{array}$ & $-0.10(0.10)$ & $0.05(0.40)$ & $<0.001$ & $-0.10(0.10)$ & $-0.10(0.10)$ & 0.441 \\
\hline $\begin{array}{l}\text { Binocular contrast sensitivity }{ }^{c} \text {, } \\
\text { median (IQR) }\end{array}$ & $1.95(0.31)$ & $1.80(0.34)$ & 0.019 & $1.95(0.30)$ & $1.95(0.30)$ & 0.943 \\
\hline Stereopsis ${ }^{\mathrm{d}}$, median (IQR) & $60(50)$ & $100(80)$ & $<0.001$ & $60(50)$ & $60(30)$ & 0.686 \\
\hline $\begin{array}{l}\text { Refraction difference between RE } \\
\text { and LE, D, median (IQR) }\end{array}$ & $0.50(0.50)$ & $1.50(1.00)$ & $<0.001$ & $0.50(0.50)$ & $0.50(0.50)$ & 0.766 \\
\hline$\geqslant 2, n(\%)$ & 0 & $41(36.0)$ & - & 0 & 0 & - \\
\hline $\begin{array}{l}\text { Visual disability (VF-14) score, } \\
\text { median (IQR) }\end{array}$ & $97.9(5.0)$ & $89.6(15.3)$ & $<0.001$ & $97.9(6.3)$ & $95.1(9.2)$ & 0.027 \\
\hline
\end{tabular}

D, Diopter; IQR, inter-quartile range; LE, left eye; logMAR, logarithm of the minimum angle of resolution; RE, right eye.

${ }^{a}$ At the first operation either both eyes (intervention group) or one eye (control group) was/were operated.

${ }^{\mathrm{b}}$ Mann-Whitney $U$-test.

'Measured by Pelli-Robson charts at $1 \mathrm{~m}$.

${ }^{\mathrm{d}}$ Measured by TNO test.

Significant $P$-values are printed in bold.

Table 4 Post-operative mean outcome measures 2 and 4 months after the last surgery ${ }^{\mathrm{a}}$ and their differences from baseline values

\begin{tabular}{|c|c|c|}
\hline Measure & ISCS: intervention, $\mathrm{n}=70$ & DSCS: control, $\mathrm{n}=114$ \\
\hline \multicolumn{3}{|l|}{ Objective measures } \\
\hline Binocular visual acuity, logMAR units, median (IQR) & $-0.10(0.10)$ & $-0.10(0.10)$ \\
\hline Difference ${ }^{\mathrm{b}}$, median (IQR) & $-0.82(0.40)$ & $-0.75(0.40)$ \\
\hline Binocular contrast sensitivity ${ }^{\mathrm{c}}$, median (IQR) & $1.92(0.31)$ & $1.95(0.30)$ \\
\hline Difference ${ }^{\mathrm{b}}$, median (IQR) & $0.67(0.30)$ & $0.75(0.30)$ \\
\hline Stereopsis $^{\mathrm{d}}$, median (IQR) & $60(50)$ & $60(32)$ \\
\hline Difference $^{\mathrm{b}}$, median (IQR) & $-250(311)$ & $-300(307)$ \\
\hline Refraction difference between RE and LE, D, median (IQR) & $0.55(0.31)$ & $0.50(0.25)$ \\
\hline Difference $^{\mathrm{b}}$, median $(\mathrm{IQR})$ & $0.12(0.62)$ & $0.12(0.62)$ \\
\hline \multicolumn{3}{|l|}{ Subjective measure } \\
\hline Visual disability (VF-14) score, median (IQR) ${ }^{\mathrm{e}}$ & $97.9(5.1)$ & $95.0(8.7)$ \\
\hline Difference $^{\mathrm{b}}$, median $(\mathrm{IQR})^{\mathrm{e}}$ & $55.6(27.2)$ & $48.7(24.7)$ \\
\hline
\end{tabular}

DSCS, delayed sequential cataract surgery; ISCS, Immediate sequential cataract surgery; IQR, inter-quartile range; logMAR, logarithm of the minimum angle of resolution.

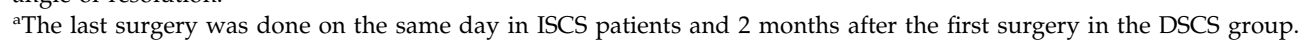

${ }^{\mathrm{b}}$ Mean post-operative values ( 2 and 4 months after the last surgery) - preoperative value.

'Measured using Pelli-Robson charts at $1 \mathrm{~m}$.

${ }^{\mathrm{d}}$ Measured using the TNO test.

e $P<0.05$ (Mann-Whitney $U$-test).

the improvement in their eyesight. However, our observed between-group difference of less than three points may not be of clinical importance. ${ }^{2}$ Lundström et $a l^{1}$ compared the ISCS and DSCS groups using the Catquest questionnaire ${ }^{28,29}$ and found that the insignificant differences in the patients' baseline selfassessed VF became significant 2 months after surgery, and then became insignificant 4 months after the last surgery. The differences in study design and in the sensitivity of the questionnaires used could account for the differences in the results between our study and the study by Lundström et al.
Smith and Liu ${ }^{11}$ classified the main concerns regarding ISCS into intraoperative and post-operative complications and then further subdivided them into catastrophic and non-catastrophic complications. Despite these concerns, ISCS has been reported to be safe in various studies. ${ }^{13-17}$ In fact, even the mortality risk from a road accident while travelling to the greater number of visits required for separate surgeries or follow-up visits was calculated to be higher than the risk of bilateral endophthalmitis in simultaneous cataract surgery. ${ }^{30}$ Similarly, ISCS was not associated with more intraoperative and post-operative complications, 
although a larger cohort of patients and a longer followup period might be needed for a conclusive answer. Refractive surprises, as a non-catastrophic complication of ISCS, were not a problem in our survey, which is compatible with previous studies. This may be due to the currently used modern biometry instrumentation and software, ${ }^{10,11,31}$ as well as the exclusion of susceptible patients ${ }^{11}$ (eg, eyes with extreme axial length or those with previous refractive surgery). In both groups, the stringent selection criteria and the strict preoperative, intraoperative, and post-operative protocols for endophthalmitis prophylaxis might have contributed to the successful surgical results without the occurrence of any serious complications. With an experienced surgeon using stringent patient selection criteria and strict aseptic protocols, ISCS safely provides greater medical, economic, and social benefits to the patients, while lowering the burden of this disease on the health-care system and society. Given the potential benefits and the limited reports of serious complications with ISCS, further studies might widen the inclusion criteria for ISCS patients.

\section{Acknowledgements}

We did not receive any financial support from any public or private sources. We have no financial or proprietary interest in a product, method, or material described herein.

\section{References}

1 Lundström M, Albrecht S, Nilsson M, Åström B. Benefits to patients of bilateral same-day cataract extraction: randomized clinical study. J Cataract Refract Surg 2006; 32: 826-830.

2 Javitt JC, Steinberg EP, Sharkey P, Schein OD, Tielsch JM, Diener $\mathrm{M}$ et al. Cataract surgery in one eye or both: a billion dollor per year issue. Ophthalmology 1995; 102: 1583-1592.

3 Castells X, Alonso J, Ribó C, Casado A, Buil JA, Badia M et al. Comparison of the results of first and second cataract eye surgery. Ophthalmology 1999; 106: 676-682.

4 Lundström M, Stenevi U, Thorburn W. Quality of life after first- and second-eye cataract surgery: five-year data collected by the Swedish National Cataract Register. J Cataract Refract Surg 2001; 27: 1553-1559.

5 Elliott DB, Patla AE, Furniss M, Adkin AE. Improvements in clinical and functional vision and quality of life after second eye cataract surgery. Optom Vis Sci 2000; 77: 13-24.

6 Laidlaw DA, Harrad RA, Hopper CD, Whitaker A, Donovan JL, Brookes ST et al. Randomised trial of effectiveness of second eye cataract surgery. Lancet 1998; 352: 925-929.

7 Castells X, Comas M, Alonso J, Espallargues M, Martínez V, García-Arumi J et al. In a randomized controlled trial, cataract surgery in both eyes increased benefits compared to surgery in one eye only. J Clin Epidemiol 2006; 59: 201-207.

8 Beatty S, Aggarwal RK, David DB, Guarro M, Jones H, Pearce JL. Simultaneous bilateral cataract extraction in the UK. Br J Ophthalmol 1995; 79: 1111-1114.

9 Ramsay AL, Diaper CJ, Saba SN, Beirouty ZA, Fawzi HH. Simultaneous bilateral cataract extraction. J Cataract Refract Surg 1999; 25: 753-762.

10 Totan Y, Bayramlar H, Cekic O, Aydin E, Erten A, Daglioglu MC. Bilateral cataract surgery in adult and pediatric patients in a single session. J Cataract Refract Surg 2000; 26: 1008-1011.

11 Smith GT, Liu CS. Is it time for a new attitude to 'simultaneous' bilateral cataract surgery? $\mathrm{Br}$ J Ophthalmol 2001; 85: 1489-1496.

12 Montan PG, Wejde G, Koranyi G, Rylander M. Prophylactic intracameral cefuroxime; efficacy in preventing endophthalmitis after cataract surgery. J Cataract Refract Surg 2002; 28: 977-981.

13 Chang DF. Simultaneous bilateral cataract surgery [editorial]. Br J Ophthalmol 2003; 87: 253-254.

14 Sarikkola AU, Kontkanen M, Kivela T, Laatikainen L. Simultaneous bilateral cataract surgery: a retrospective surgery. J Cataract Refract Surg 2004; 30: 1335-1341.

15 Johnsson BA, Lundh BL. Bilateral same day phacoemulsification: 220 cases retrospectively reviewed. $\mathrm{Br}$ J Ophthalmol 2003; 87: 285-290.

16 Wertheim M, Burton R. Immediately sequential phacoemulsification performed under topical anesthesia as day case procedure. Br J Ophthalmol 2002; 86: 1356-1358.

17 Arshinoff SA, Strube YN, Yagev R. Simultaneous bilateral cataract surgery. J Cataract Refract Surg 2003; 29: 1281-1291.

18 Desai P, Reidy A, Minassian DC, Vafidis G, Bolger J. Gains from cataract surgery: visual function and quality of life. $\mathrm{Br}$ J Ophthalmol 1996; 80: 868-873.

19 Uusitalo RJ, Tarkkanen A. Outcomes of small incision cataract surgery. J Cataract Refract Surg 1998; 24: 212-221.

20 Seal DV, Barry P, Gettinby G, Lees F, Peterson M, Revie CW, et al., ESCRS Endophthalmitis Study Group. ESCRS study of prophylaxis of postoperative endophthalmitis after cataract surgery: Case for a European multicenter study. J Cataract Refract Surg 2006; 32: 396-406.

21 Pelli DG, Robson JG, Wilkins AJ. The design of a new letter chart for measuring contrast sensitivity test. Clin Vis Sci 1987; 88: 2187-2199.

22 Mäntyjärvi M, Laitinen T. Normal values for the PelliRobson contrast sensitivity test. J Cataract Refract Surg 2001; 27: 261-266.

23 Williamson TH, Strong NP, Sparrow J, Aggarwal RK, Harrad R. Contrast sensitivity and glare in cataract using the Pelli-Robson chart. Br J Ophthalmol 1992; 76: 719-722.

24 Steinberg EP, Tielsch JM, Schein OD, Javitt JC, Sharkey P, Cassard SD et al. The VF-14: an index of functional impairment in patients with cataract. Arch Ophthalmol 1994; 112: 630-638.

25 Cassard SD, Patrick DL, Damiano AM, Legro MW, Tielsch JM, Diener-West $\mathrm{M}$ et al. Reproducibility and responsiveness of the VF-14: an index of functional impairment in patients with cataracts. Arch Ophthalmol 1995; 113: 1508-1513.

26 Alonso J, Espallargues M, Andersen TF, Cassard SD, Dunn E, Bernth-Petersen P et al. International applicability of the VF-14: an index of visual function in patients with cataracts. Ophthalmology 1997; 104: 799-807. 
27 Talbot EM, Perkins A. The benefit of second eye cataract surgery. Eye 1998; 12: 983-989.

28 Lundström M, Roos P, Jensen S, Fregell G. Catquest questionnaire for use in cataract surgery care: description, validity, and reliability. J Cataract Refract Surg 1997; 23: 1226-1236.

29 Lundström M, Stenevi U, Thorburn W, Roos P. Catquest questionnaire for use in cataract surgery care: assessment of surgical outcomes. J Cataract Refract Surg 1998; 24: 968-974.

30 Ellis MF, Bolger J, Steinmetz B, Claoué C. Responses to December 1997 consultation section [letters]. J Cataract Refract Surg 1998; 24: 430-432.

31 Holladay JT. Standardizing constants for ultrasonic biometry, keratometry, and intraocular lens power calculations. J Cataract Refract Surg 1997; 23: 1356-1370. 